\title{
Aspectos epidemiológicos e clínicos do escorpionismo na região de Santarém, Estado do Pará, Brasil
}

\author{
Epidemiological and clinical aspects of scorpion envenomation \\ in the region of Santarém, Pará, Brazil
}

\author{
Pedro Pereira de Oliveira Pardal' ${ }^{1}$, Lívia Correa Castro ${ }^{2}$, Erik Jennings ${ }^{2}$, Joseana Silva de \\ Oliveira Pardal' e Maria Rita de Cássia da Costa Monteiro'
}

\begin{abstract}
Resumo Este trabalho é um estudo prospectivo e descritivo dos aspectos epidemiológicos e clínicos de 72 envenenamentos por escorpiões admitidos no Hospital Municipal de Santarém, Estado do Pará, Brasil, entre fevereiro de 2000 a fevereiro de 2001. Trouxeram o animal 8,3\% das vítimas, os quais foram identificados como T. cambridgei. O sexo masculino foi acometido em $83,3 \%$. A idade das vítimas e o tempo para o socorro médico foram respectivamente de 33,6 18,3 anos e 4,6 3,2 horas em média. Os membros superiores foram acometidos em $51,5 \%$ dos casos. As manifestações locais estiveram presentes em $91,7 \%$ e as sistêmicas em 98,6\% dos envenenamentos. Entre os sintomas locais encontramos: parestesia em 79,2\%, dor em 52,8\%, e edema em $26,4 \%$ dos casos. Nas manifestações sistêmicas predominou as queixas neurológicas em $97,2 \%$ das vítimas, sendo o sintoma de sensação de "choque elétrico" pelo corpo (88,9\%) o mais freqüente. No exame neurológico os sinais mais encontrados foram: mioclonias (93,0\%), dismetria (86,1\%), disartria (80,6\%) e ataxia de marcha (70,8\%). Classificou-se como moderados $76,4 \%$ dos envenenamentos, sem nenhum caso grave. Deixaram de realizar a soroterapia 32,7\% dos casos moderados, por ausência de soro específico no momento do atendimento. O escorpionismo da região de Santarém mostra um comportamento clínico regional diferente daqueles descritos no Brasil e de outros locais da Amazônia e, apresenta uma clínica predominantemente neurológica, ainda não descrita na literatura brasileira.
\end{abstract}

Palavras-chaves: Escorpionismo. Tityus cambridgei. Neurológico. Santarém.

\begin{abstract}
This is a descriptive and prospective study on epidemiological and clinical aspects of 72 scorpion accidents admitted to Santarém Municipal Hospital, state of Pará, Brazil, from February 2000 to February 2001. Only 8.3\% brought the animal with them, identified as T. cambridgei. The majority of victims were male (83.3\%). The mean age and the time of the medical help were respectively $33.6 \pm 18.3$ years and $4.6 \pm 3.2$ hours. The parts of the body most affected were the superior members (51.5\%). Local symptoms occurred in $91.7 \%$ cases and systemic manifestations in $98.6 \%$ of the accidents. The local symptoms included: paresthesia in $79.2 \%$ cases, pain in $52.8 \%$ and edema in $26.4 \%$. Among the systemic manifestations neurological disorders predominated in $97.2 \%$, and the symptom of "electric shock" occurred in $88.9 \%$ patients. The most common neurological signs were: myoclonia (93\%), dysmetria (86.1\%), dysarthria (80.6\%), and ataxia (70.8\%). The accidents were classified as moderate in $76.4 \%$ without any serious cases. The specific anti-venom serum was not administered in $32.7 \%$ of the moderate cases, due to non-availability of the anti-venom serum at the time of attendance. The victims of scorpion envenomation notified at Santarém, present a different clinical and regional behavior from previous reports in Brazil and Amazonia regions. The predominantly neurological picture has not previously been described in the Brazilian literature.
\end{abstract}

Key-words: Scorpionism. Tityus cambridgei. Neurological. Santarém.

\footnotetext{
1. Disciplina de Doenças Infecciosas e Parasitárias do Departamento de Patologia Tropical do Centro de Ciências da Saúde da Universidade Federal do Pará, Belém, PA. 2. Hospital Municipal de Santarém, Pará.

Endereço para correspondência: Dr. Pedro Paulo Oliveira Pardal. Hospital Universitário João de Barros Barreto/Centro de Informações Toxicológicas de Belém. Rua dos Mundurucus 4487, Guamá, 66073-000 Belém, PA, Brasil.

Tel: 91 249-6370; Fax: 91259.3748

e-mail: citbelem@yahoo.com

Recebido em 16/7/2001

Aceito em 27/3/2003
} 
O escorpionismo é um importante problema de saúde pública para vários países tropicais e subtropicais, como o Brasili3 ${ }^{13}$, México ${ }^{15} 1820$, Tunísia e Marrocos ${ }^{18}$, África do Sul ${ }^{21}$, Egito ${ }^{12}$ e Estados Unidos da América ${ }^{4}$.

Os escorpiões possuem glândulas de veneno, mas somente cerca de 25 das 1.400 espécies conhecidas são consideradas perigosas, podendo levar o acidentado ao óbito ${ }^{18}$.

Os principais agentes de importância médica no Brasil são o T. serrulatus, T. bahiensis e T. stigmurus, e $50 \%$ das notificações são procedentes de S. Paulo e Minas Gerais ${ }^{13}$. O T. serrulatus, de maior freqüência na América do Sul, é o responsável pelos acidentes com maior gravidade 6710111327 . Na Amazônia, os mais encontrados são o T. cambridgei, T. silvestris e o T. metuendus, entre outros ${ }^{13161723}$. Lourenço ${ }^{17}$ em 1984, refere que nas regiões de Santarém-Trombetas os escorpiões encontrados nas matas são o T. metuendus, T. silvestris, entre outros.

Os primeiros estudo sobre o veneno de escorpiões e do envenenamento no Brasil, foram realizados por Murano $^{22}$ em 1915 e Vital Brazil ${ }^{5}$ em 1918. O veneno possui uma mistura complexa de aproximadamente 70 aminoácidos, que possui similaridade de composição, nas diferentes espécies ${ }^{1}$, e suas manifestações clínicas estão na predominância dos efeitos simpáticos ou parassimpáticos, em decorrências da liberação de catecolaminas e acetilcolinas pelas terminações nervosas pós-ganglionares ${ }^{113}$ cujos efeitos serão de neurotoxidade e cardiotoxidade ${ }^{20}$.

$\mathrm{Na}$ Amazônia brasileira, referências sobre o escorpionismo são encontradas em vários trabalhos. Menezes et $\mathrm{al}^{19}$, que referem ser o escorpião o artrópode que mais ocasionou acidentes nos pacientes atendidos pelo Centro de Informações Toxicológicas de Belém; Yamano et $\mathrm{al}^{30}$, informam ser ele a $2^{\mathrm{a}}$ causa entre os animais peçonhentos. Asano et $\mathrm{al}^{3}$ descreveram doze acidentes leves na área metropolitana de Belém e arredores $^{23}$ sendo o $T$. silvestris identificado como o responsável por dois destes casos. Acidentes fora destes locais, encontram-se os referidos em Itaituba na região do Tapajós, Pará ${ }^{26}$ e em Manaus, Amazonas ${ }^{29}$.

O quadro clínico do escorpionismo é semelhante nas diversas regiões do Brasil $^{36131427}$, e está na dependência da gravidade do acidente. Constitui-se principalmente de manifestações locais e autonômicas, com discreto ou nenhum comprometimento neurológico 6142627 .

Com o objetivo de conhecer os aspectos epidemiológico e clínico do envenenamento por escorpiões da região de Santarém, é que foi realizado este estudo nas vítimas admitidas no Hospital Municipal de Santarém (HMS), Pará, Brasil.

\section{CASUÍSTICA E MÉTODOS}

Foram estudados prospectivamente e descritivamente 72 acidentes por escorpiões admitidos, no período de fevereiro de 2000 a fevereiro de 2001, no Hospital Municipal de Santarém.

Santarém é município do Estado do Pará, Brasil, localizado a margem direita do Rio Tapajós, na confluência do Rio Amazonas, e distante $1.369 \mathrm{~km}$ de Belém, capital do Estado, possuindo 10 municípios limítrofes.
Durante o atendimento, os autores deste estudo, seguindo um protocolo, realizaram inquérito clínico, epidemiológico e propedêutica neurológica das vítimas. Nenhum exame laboratorial complementar foi realizado.

Os escorpiões causadores dos envenenamentos e trazidos pelos pacientes foram identificados no laboratório de artrópodes do Instituto Butantan de São Paulo.

\section{RESULTADOS}

Os 72 acidentes por escorpiões atendidos no Hospital Municipal de Santarém, tiveram como procedência Santarém $(94,4 \%)$, Belterra $(4,2 \%)$ e Prainha $(1,4 \%)$, estes dois, municípios limítrofes do local onde foram socorridos. A maior incidência ocorreu nos meses de março e agosto (Tabela 1). O sexo masculino foi o mais acometido (83,3\%). A idade das vítimas variou de 02 a 74 anos, com média de 33,6 $\pm 18,3$ anos. Até 14 anos de idade estavam $13 \%$ dos acidentados; $84,1 \%$ entre 15 a 65 e 2,8\% encontravam-se acima de 65 anos.

O escorpião foi denominado de lacrau entre $87,5 \%$ dos acidentados; de escorpião $4,2 \%$, e não souberam identificar 8,3\%. Informaram ser preto o animal agressor $5,6 \%$ das vítimas; preto e grande em $59,7 \%$; amarelo em $1,4 \%$, e não souberam informar $33,3 \%$. Trouxeram o animal causador do acidente $8,3 \%$ dos indivíduos estudados, todos procedentes do município de Santarém, e foram identificados como T. cambridgei.
A maioria $(81,9 \%)$ dos acidentes ocorreu no período diurno, sendo $40,3 \%$ pela manhã e $41,6 \%$ à tarde. Entre 19 horas e 6 horas ocorreram 18,1\% dos casos. O tempo entre o acidente e o socorro médico variou de 30 minutos a 14 horas, com uma média de 4,6 $\pm 3,2$ horas.

O membro superior foi acometido em $51,5 \%$ dos casos, e a mão em 41,1\%; o membro inferior sofreu agressão em $43,1 \%$ das vítimas, e o pé em $38,9 \%$ das vezes. Outras partes do corpo foram atingidas em $5,4 \%$ dos acidentes.

Apresentaram sintomas e sinais locais $91,7 \%$ das vítimas (Tabela 2) e manifestações clínicas sistêmicas $98,6 \%$ dos envenenamentos (Tabela 3 ).

Na clínica neurológica, foram mais comuns os sintomas de sensação de choque elétrico pelo corpo (88,9\%). Os sinais mais observados foram as mioclonias (93\%), dismetria $(86,1 \%)$, disartria $(80,6 \%)$ e ataxia de marcha $(70,8 \%)$ dos casos. 
Tabela 1 - Meses dos acidentes por escorpiões em SantarémPará, Brasil.

\begin{tabular}{lrr}
\hline Mês & № & $\%$ \\
\hline Janeiro & - & - \\
Fevereiro & 7 & 9,7 \\
Março & 13 & 18,1 \\
Abril & 8 & 11,1 \\
Maio & 6 & 8,3 \\
Junho & - & - \\
Julho & 5 & 6,9 \\
Agosto & 13 & 18,1 \\
Setembro & 6 & 8,3 \\
Outubro & - & - \\
Novembro & 7 & 9,7 \\
Dezembro & 5 & 6,9 \\
Janeiro & - & - \\
Fevereiro & 2 & 2,8 \\
\hline Total & 72 & 100,0 \\
\hline
\end{tabular}

Os pacientes ainda apresentaram outras manifestações neurológicas, quando do exame neurológico, mas que, não foram contempladas na tabela, como: reflexos profundos sincinéticos $(9,7 \%)$, disdiadococinesia $(7 \%)$, confusão mental $(4,2 \%)$, reflexos profundos policinéticos $(2,8 \%)$ e alucinações visuais $(1,4 \%)$.

Os envenenamentos foram classificados como leves $(23,6 \%)$ ou moderados $(76,4 \%)$, porém, o antiveneno não foi realizado em $32,7 \%$ dos casos moderados, por falta do soro específico no hospital durante o atendimento médico. Foram usadas $63,6 \%$ de soro antiescorpiônico

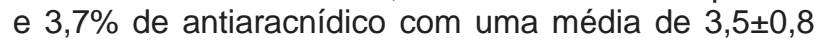
ampolas. Todos os pacientes receberam alta, curados, sendo que em $98,6 \%$ dos casos ela ocorreu em 24 horas e em apenas 1,4\%, em 03 dias.

\section{DISCUSSÃO}

A denominação de lacrau é também freqüente em outras regiões do país ${ }^{13}$. Os poucos animais agressores levados ao hospital em sua totalidade, foram identificados como T. cambridgei. Muitos foram caracterizados como sendo de cor preta ou preta e grande, cor esta própria do T. cambridgei e do T. metuendus, quando adultos ${ }^{16}$. Embora o T. cambridgei seja a espécie mais freqüente na Amazônia Ocidental, quase não existem registros de acidentes com estes agente $^{313}$.

Considerando-se as condições climáticas, os acidentes por escorpião ocorrem no sudeste do país nos meses quentes e chuvosos ${ }^{13}$. Na Bahia, de junho a setembro ${ }^{14}$, São Paulo de outubro e fevereiro ${ }^{27}$ e Uberlândia em outubro e novembro ${ }^{28}$. No presente estudo, não se observou nenhuma relação com as variações climáticas da região, pois tivemos uma maior incidência nos meses de março, época em que se tem o maior índice pluviométrico e agosto, quando é
Tabela 2 - Os sintomas e sinais locais no escorpionismo de Santarém, Pará

\begin{tabular}{lrr}
\hline Sintomas/sinais & № & $\%$ \\
\hline Parestesia & 57 & 79,2 \\
Dor & 38 & 52,8 \\
Edema & 19 & 26,4 \\
Eritema & 7 & 9,7 \\
Calor & 3 & 4,2 \\
Equimose & 1 & 1,4 \\
Endurado & 1 & 1,4 \\
Piloereção & 1 & 1,4 \\
Dor região inguinal & 1 & 1,4 \\
Nenhum & 6 & 8,3 \\
\hline
\end{tabular}

Tabela 3 - Manifestações clínicas sistêmicas no escorpionismo da região de Santarém, Pará.

\begin{tabular}{lrr}
\hline Manifestações clínicas & $\mathrm{n}^{\circ}$ & $\%$ \\
\hline Manifestações gerais & 5 & 6,9 \\
prostração & 2 & 2,8 \\
tremores & 2 & 2,8 \\
astenia & 1 & 1,4 \\
hipertermia & 1 & 1,4 \\
Manifestações autonômicas & 30 & 41,6 \\
taquicardia & 14 & 19,4 \\
sudorose & 13 & 18,1 \\
sialorréia & 6 & 8,3 \\
náusea & 6 & 8,3 \\
hipertensão arterial & 6 & 8,3 \\
vômitos & 4 & 5,6 \\
Manifestações neurológicas & 70 & 97,2 \\
sintomas neurológicos & & \\
$\quad$ sensação de "choque elétrico" pelo corpo & 64 & 88,9 \\
$\quad$ sensação de parestesia em todo corpo & 36 & 50,0 \\
sinais neurológicos & & \\
mioclonias & 67 & 93,0 \\
dismetria & 62 & 86,1 \\
disartria & 58 & 80,6 \\
ataxia de marcha & 71 \\
$\quad$ hiperreflexia & 51 & 70,8 \\
$\quad$ espasticidade & 41 & 56,9 \\
$\quad$ hiperalerta & 25 & 34,7 \\
romitação & 29,2 \\
$\quad$ sonolento & 26,4 \\
babinski & 19 & 12,3 \\
\hline & & 9,7 \\
\hline
\end{tabular}

pequena esta precipitação, entretanto o período de observação foi curto.

Segundo a literatura ${ }^{27}$, é no período diurno que ocorrem a maioria dos acidentes no Brasil. Resultados semelhantes foram constatados neste trabalho.

O sexo masculino foi o mais acometido no presente estudo, semelhante ao que se observou em São Paulo27. Em Belém, Pará30, ocorre predominância do feminino, enquanto em Uberlândia ${ }^{28}$ e na Região do TapajósPará26, não há predominância de sexo. 
A idade média das vítimas $(33,6 \pm 18,3$ anos) difere da encontrada em Itaituba, região Oeste do Pará, onde a média foi de $23,1 \pm 16,4$ anos $^{26}$. Em Belém-Pará foram os menores de 20 anos os mais acidentados ${ }^{30}$, enquanto em São Paulo e Uberlândia a faixa etária mais acometida é a de 20 a 30 anos $^{27} 28$.

O membro superior foi o mais acometido. Resultados semelhantes da literatura brasileira ${ }^{732728}$ e mexicana ${ }^{15}$, porém na África o Sul ${ }^{21}$ e no Egito ${ }^{12}$, são os membros inferiores os mais acidentados.

O tempo para o atendimento dos pacientes, no presente estudo, foi de 4,6 \pm 3.2 horas, maior do que o observado em Itaituba ${ }^{26}$, no Pará. Em São Paulo ${ }^{27}$ e Uberlândia ${ }^{28}$, mais da metade das vítimas são atendidas nas primeiras duas e três horas do acidente.

A maioria dos acidentes no Brasil são classificados como leve ${ }^{3} 13142730$, e nos casos graves a letalidade chega a $0,58 \%{ }^{13}$, sendo o óbito associado com maior freqüência ao $T$. serrulatus ${ }^{61113}$. No Nordeste existem relatos de óbitos por T. stigmurus ${ }^{10}{ }^{14}$. Nos nossos achados, apesar dos pacientes apresentarem uma exuberante manifestação clínica, não encontramos nenhum caso grave, consequentemente nenhum óbito. A soroterapia específica foi realizada em $67,3 \%$ dos casos moderados, enquanto nos outros apenas sintomáticos, isto em decorrência da falta do antiveneno no momento do atendimento, mas mesmo assim todos pacientes evoluíram para a cura.

As manifestações clínicas locais do escorpionismo brasileiro ${ }^{36814272830}$ e de outros países 49121521 são semelhantes aos nossos achados.

As manifestações sistêmicas estiveram presentes em 98,6\%, dos pacientes, predominando a clínica neurológica sobre as autonômicas e gerais. O que difere dos envenenamentos por escorpiões encontrados no restante do país, onde as manifestações autonômicas e gerais predominam, com poucas ou nenhuma alterações para o lado do sistema nervoso central 68101314272830 .

Quando se realizou o exame da motricidade, para avaliar a coordenação, a maioria dos pacientes apresentou dismetria. $\mathrm{Na}$ motricidade automática, tivemos ataxia de marcha, e na motricidade involuntária, observamos mioclonias, sendo que as reflexas estiveram presentes em $86,5 \%$, e o estímulo sensitivo desencadeou as mesmas em $73,6 \%$.

Manifestações clínicas neurológicas de mioclonias são traduzidas pelos pacientes como sensação de choque pelo corpo, os quais foram descritos pela primeira vez em Itaituba, Pará ${ }^{6}$. Outros sintomas como dificuldade de deambulação, contratura e contração muscular são citadas na literatura nacional ${ }^{814}$. No México, as manifestações neurológicas são referidas como fala arrastada, contrações musculares ${ }^{20}$ e disartria ${ }^{15}$. Na África do Sul referem disartria e movimentos involuntários ${ }^{21}$. No Egito citam mioclonias ${ }^{12}$ e nos Estados Unidos da América referem movimentos de contração das extremidades ${ }^{4}$.

A presença de manifestações neurológicas exuberantes, como mioclonias, dismetria, disartria, e ataxia de marcha entre outras, na vigência de clínica autonômica e geral discreta, nos induz a admitir tratar-se de um comportamento clínico regional do escorpionismo, em relação a outras regiões do Brasil, incluindo a Amazônia, ainda não descrito na literatura brasileira.

\section{AGRADECIMENTOS}

A equipe de saúde do Hospital Municipal de Santarém, pela colaboração durante a coleta de dados. A Dra. Denise Maria Cândido do laboratório de artrópodes do Instituto Butantan de São Paulo, pela identificação dos animais e, ao Dr. Bernardo Cardoso, pelo apoio durante nossa permanência em Santarém.

\section{REFERÊNCIAS BIBLIOGRÁFICAS}

1. Amitai Y. Clinical manifestation and management of scorpion envenomation. Public Health Reviews 26:257-263, 1998.

2. Araújo FAA, Resende CC. Escorpionismo no Brasil, 1988-1989. Fundação Nacional de Saúde/Ministério da Saúde/Programa Nacional de Ofidísmo. Brasília p. 47, 1990.

3. Asano ME, Arnund RM, Lopes FOB, Pardal JSO, Pardal PPO. Estudo Clínico e epidemiológico de 12 acidentes por escorpiões atendidos no Hospital Universitário João de Barros Barreto, Belém-Pará, no período de 1992-1995. Revista da Sociedade Brasileira de Medicina Tropical 29(Supl.I):243, 1996.

4. Bond GR. Snake, spider, and scorpion envenomation in North America. Pediatrics in Review 20:147-150, 1999.

5. Brazil V. Contribuição ao estudo do envenenamento pela picada do escorpião e seu ratamento pelo Dr. Vital Brazil. In: Collectanea de Trabalhos. Instituto Butantan (1901-1917). Typografia do Diário Oficial de São Paulo p. 69-81, 1918.

6. Bucaretchi F, Baracat ECE, Nogueira RJN, Chaves A, Zambrone FAD, Fonseca MRCC, Tourinho FS. A comparative study of severe scorpion envenomation in children caused by Tityus bahiensis and Tityus serrulatus. Revista do Instituto de Medicina Tropical de São Paulo 37:331-336, 1995

7. Bücherl W. Escorpionismo no Brasil. Memórias do Instituto Butantan 34:9-24, 1969.

8. Cupo P, Jurca M, Azevedo-Marques MM, Oliveira JSM, Hering SE. Severe scorpion envenomation in Brazil. Clinical, laboratory and anatomicopathological aspects. Revista do Instituto de Medicina Tropical de São Paulo 36:67-76, 1994.

9. El Naggar MK, Wahib AA, Montasser MF. Clinical patterns of scorpion stings in Saudi Arabia. Journal of the Egyptian Society of Parasitology 15:133-38, 1985. 
10. Eickstedt VRDV. Escorpionismo por Tityus stigmurus no Nordeste do Brasil (Scorpiones; Buthidae). Memórias do Instituto Butantan 47/48:133-137, 1983/1984.

11. Eickstedt VRD, Ribeiro LA, Candido DM, Albuquerque MJ, Jorge MT. Evolution of scorpionism by Tityus bahiensis (Perty) and Tityus serrulatus Lutz and Mello and geographical distribution of the two species in the state of São Paulo - Brazil. Journal of Venomous Animals and Toxins 2:92-105, 1996.

12. Farghly WMA, Ali FA. A clinical and neurophysiological study of scorpion envenomation in Assiut, upper Egypt. Study of scorpion envenomation in Assiut, upper Egypt. Acta PaediatricInternational Journal of Paediatrics 88:290-294, 1999.

13. Fundação Nacional de Saúde. Manual de Diagnóstico e Tratamento de Acidentes por Animais Peçonhentos. Ministério da Saúde, Brasília, p. 141, 1998.

14. Lira-da-Silva RM, Amorim AM, Brazil TK. Envenenamento por Tityus stigmurus (Scorpiones; Buthidae) no Estado da Bahia, Brasil. Revista da Sociedade Brasileira de Medicina Tropical 33:239-245, 2000.

15. Lomelí AH, Osronio IR, Cabrera RC. Prevalência de la picadura de alacrán em la ciudad de Querétaro. Revista Médica del Instituto Mexicano del Seguro Social 32:527-531, 1994.

16. Lourenço WR. Revisão crítica das espécies Tityus no Estado do Pará (Scorpiones, Butidae). Boletim do Museu Paraense Emílio Goeldi Zoologia 1:5-18, 1984.

17. Lourenço WR. Sinopse da fauna escorpiônica no Estado do Pará, especialmente as regiões de Carajás, Tucuruí, Belém e Trombetas. Boletim do Museu Paraense Emílio Goeldi, Zoologia 4:155-173, 1984.

18. Lourenço WR, Cuellar O. Scorpions, Scorpionism, life history strategies and parthenogenesis. The Journal of Venomous Animals and Toxins 1:51-62, 1995.

19. Menezes KP, Souza JB, Guimarães AC, Almeida RG, Magalhães AFA, Pardal PPO. Acidentes por artrópodes notificados e orientados pelo CIT-Belém no período de 30/3/1998 a 31/12/ 1999. Revista da Sociedade Brasileira de Medicina Tropical 33(supl I):161, 2000.

20. Montoya-Cabrera MA. Alacranismo. Gaceta Médica de México 132:645-648, 1996.
21. Müller GJ. Scorpionism in South Africa. A report of 42 serious scorpion envenomations. South African Medical Journal 83:405411, 1993.

22. Murano HR. Do escorpionismo. Tese de Doutorado. Faculdade de Medicina do Rio de Janeiro, RJ, 1915.

23. Neto HF, Pardal PPO. Escorpiões e escorpionismo na área metropolitana de Belém e arredores, Estado do Pará, Brasil. (Chelicerata, Scorpiones). Anais da Academia de Medicina do Pará, Belém 7:31-35, 1996.

24. Nishioka SA, Silveira PVP, Ugrinovich R, Oliveira RB. Letter to the editor. Scorpion sting with cranial nerve involvement. Toxicon 30: 685-686, 1992.

25. Nishioka SA, Silveira PVP, Pereira CAD. Scorpion sting on the penis. The Journal of Urology 150:1501, 1993.

26. Pardal PPO, Cardoso BS, Fan HW. Escorpionismo na região do rio Tapajós, Itaituba (Pará). Revista da Sociedade Brasileira de Medicina Tropical 32 (supl I):394, 1999.

27. Ribeiro AL, Rodrigues L, Jorge MT. Aspectos clínicos e epidemiológicos do envenenamento por escorpiões em São Paulo e municípios próximos. Revista de Patologia Tropical 30: 83-92, 2001.

28. Silva FG, Queiroz FM, Ribeiro LB, Ribeiro LA, Borges PP, Motta RF, Spina VP, Assunção VF. Acidentes por escorpião: avaliação epidemiológica e clínica de 764 casos do HC-UFU de 1987 a 1996. Revista da Sociedade Brasileira de Medicina Tropical 32(supl I):385, 1999.

29. Souza ARB, Arakian SKL, Buhrnhein PF. Estudo clínico epidemiológico dos acidentes escorpiônicos atendidos no Instituto de Medicina Tropical de Manaus, no período de 1986 a 1994. Revista da Sociedade Brasileira de Medicina Tropical 28 (supl I):167, 1995.

30. Yamano EYS, Hidaka ASV, Negrão SG, Souza N, Lima SGL, Souza ZN, Magalhães AA, Miranda JBB, Esteves FAL, Vieira JL, Pardal PPO. Aspectos epidemiológicos e Clínicos dos acidentes por escorpiões orientados pelo Centro de Informações Toxicológicas de Belém, no período de maio de 1997 a novembro de 1998. Revista da Sociedade Brasileira de Medicina Tropical 32(supl I):394, 1999. 